\title{
Matrix Membranes and Integrability
}

\author{
Cosmas Zachos $^{1}$, David Fairlie ${ }^{2}$, and Thomas Curtright ${ }^{3}$ \\ ${ }^{1}$ High Energy Physics Division, Argonne National Laboratory, Argonne, \\ IL 60439-4815, USA zachos@hep.anl.gov \\ 2 Department of Mathematical Sciences, University of Durham, Durham, \\ DH1 3LE, UK David.Fairlie@durham.ac.uk \\ ${ }^{3}$ Department of Physics, University of Miami, Box 248046, Coral Gables, \\ FL 33124, USA curtright@phyvax.ir.Miami.edu
}

\begin{abstract}
This is a pedagogical digest of results reported in [Curtright, Fairlie, \& Zachos 1997], and an explicit implementation of Euler's construction for the solution of the Poisson Bracket dual Nahm equation. But it does not cover 9 and 10-dimensional systems, and subsequent progress on them [Fairlie 1997]. Cubic interactions are considered in 3 and 7 space dimensions, respectively, for bosonic membranes in Poisson Bracket form. Their symmetries and vacuum configurations are explored. Their associated first order equations are transformed to Nahm's equations, and are hence seen to be integrable, for the 3-dimensional case, by virtue of the explicit Lax pair provided. Most constructions introduced also apply to matrix commutator or Moyal Bracket analogs.
\end{abstract}

\section{Introduction}

A proposal for non-perturbative formulation of M-theory [Banks et al. 1997] has encouraged a reappraisal of matrix membrane theory [Collins \& Tucker 1976, Hoppe 1982]. Symmetry features of membranes and their connection to matrix models [Hoppe 1982, Floratos et al. 1989, Fairlie, Fletcher, \& Zachos 1989, Floratos 1989, Fairlie, Fletcher, \& Zachos 1990] have been appreciated for quite some time. Effectively, infinite- $N$ quantum mechanics matrix models (presented as a restriction of $\mathrm{SU}(\infty)$ Yang-Mills theories) amount to membranes, by virtue of the connection between $\mathrm{SU}(N)$ and area-preserving diffeomorphisms (SDiff) generated by Poisson Brackets: in these, "color" algebra indices Fouriertransform to "membrane" sheet coordinates. The two are underlain and linked by Moyal Brackets, the universal associative generalization of Poisson Brackets.

Below, we care to introduce novel Poisson Bracket interactions for a bosonic membrane embedded in 3-space

$$
\mathcal{L}_{I P B}=\frac{1}{3} \epsilon^{\mu \nu \kappa} X^{\mu}\left\{X^{\nu}, X^{\kappa}\right\}
$$

which are restrictions of the Moyal Bracket generalization

$$
\mathcal{L}_{I M B}=\frac{1}{3} \epsilon^{\mu \nu \kappa} X^{\mu}\left\{\left\{X^{\nu}, X^{\kappa}\right\}\right\}
$$


which, in turn, also encompasses the plain matrix commutator term

$$
\mathcal{L}_{I C}=\frac{1}{3} \epsilon^{\mu \nu \kappa} X^{\mu}\left[X^{\nu}, X^{\kappa}\right] .
$$

The structure of (1) may be recognized as that of the interaction term $\epsilon_{i j l} \phi^{i} \epsilon^{\mu \nu} \partial_{\mu} \phi^{j} \partial_{\nu} \phi^{j}$ of the 2-dimensional SO(3) pseudodual chiral $\sigma$-model of Zakharov \& Mikhailov (1978) - this is a limit of the WZWN interaction term, where the integer WZWN coefficient goes to infinity while the coupling goes to zero, such that the product of the integer with the cube of the coupling is kept constant [Curtright \& Zachos 1995]. (N.B. Contrast to the interaction of a different model [Plebanski et al. 1996], with derivative structure 0-2-2, which could be regarded as a large- $\mathrm{N}$ limit of the pseudodual interaction exemplified above by $\mathrm{SO}(3)$.)

Alternatively, the structure of (3) is linked to what remains of the gauge theory instanton density,

$$
K^{0}=\epsilon^{\mu \nu \kappa} \operatorname{Tr} A_{\mu}\left(\partial_{\nu} A_{\kappa}-\frac{1}{4}\left[A_{\nu}, A_{\kappa}\right]\right),
$$

in the standard space-invariant limit (where the first term vanishes).

There is some formal resemblance to membrane interaction terms introduced in Zaikov (1991) (in that case a quartic in the $X^{\mu_{\mathrm{S}}}$ ), which, in turn, reflect the symplectic twist of topological terms of Biran et al. (1987) for self-dual membranes. But, unlike those interactions, the cubic terms considered here do not posit full Lorentz invariance beyond 3-rotational invariance: they are merely being considered as quantum mechanical systems with internal symmetry. One may therefore expect this fact to complicate supersymmetrization.

We also succeed in introducing analogous trilinear interactions for membranes embedded in 7-space, which evince similarly interesting properties.

In what follows, after a brief review of some matrix membrane technology, we explore the symmetry features of the new terms, and the remarkable symmetry of the corresponding vacuum configurations; we describe classical configurations of the Nahm type, which we find to be integrable in $3 \mathrm{~d}$, as in the conventional membrane models. When we interchange the rôle of dependent and independent variables of the 3d PB Nahm equations, we detail how these "dual" equations are solved by Euler's construction, based on harmonic scalar functions. Our discussion will concentrate on Poisson Brackets, but the majority of our results carry over to the Moyal Bracket and matrix commutator cases, by dint of the underlying formal analogy.

However, even though mostly integrable first-order equations are studied here, it should be borne in mind that the behaviour of the generic solutions to the second-order equations of motion for such systems is often chaotic. For example, in the case of QM matrix models (Yang-Mills on a finite gauge group, with fields dependent only upon time), characteristic features of chaotic behaviour were observed on the solutions of the second-order equations of motion 
[Matinyan et al. 1981]. Still, it is not known whether this ergodicity persists in the large $N$ limit, i.e. the corresponding PB system.

Subtler topological considerations of special features for various membranes are not addressed here. Nontrivial boundary terms, e.g. of the type linked to D-branes, are also not considered.

\section{Review of Brackets, Matrix Commutators, and Matrix Membrane Actions}

Poisson Brackets, Moyal Brackets, and matrix commutators are inter-related antisymmetric derivative operations, sharing similar properties, such as integration by parts, associativity (hence comportance to the Jacobi identity), suitable Leibniz chain rules, etc. They are all representable as commutators of associative operators. Much of their technology is reviewed in [Moyal 1949; Fairlie, Fletcher, \& Zachos 1989; Fairlie, Fletcher, \& Zachos 1990; Hoppe 1990].

Poisson Brackets act on the "classical phase-space" of Fourier-transformed color variables, with membrane coordinates $\xi=\alpha, \beta$,

$$
\left\{X^{\mu}, X^{\nu}\right\}=\frac{\partial X^{\mu}}{\partial \alpha} \frac{\partial X^{\nu}}{\partial \beta}-\frac{\partial X^{\mu}}{\partial \beta} \frac{\partial X^{\nu}}{\partial \alpha} .
$$

This may be effectively regarded as the infinitesimal canonical transformation on the coordinates $\xi$ of $X^{\nu}$, generated by $\nabla X^{\mu} \times \nabla$, s.t. $(\alpha, \beta) \mapsto$ $\left(\alpha-\partial X_{\mu} / \partial \beta, \beta+\partial X_{\mu} / \partial \alpha\right)$, which preserves the membrane area element $d \alpha d \beta$. This element is referred to as a symplectic form and the class of transformations that leaves it invariant specifies a symplectic geometry; the area preserving diffeomorphisms are known as SDiff.

PBs correspond to $N \rightarrow \infty$ matrix commutators [Hoppe 1982]. However, there is a generalization which covers both finite and infinite $N$. The virtually unique associative generalization of PBs is the Moyal Bracket [Moyal 1949],

$$
\left\{\left\{X^{\mu}, X^{\nu}\right\}\right\}=\left.\frac{1}{\lambda} \sin \left(\lambda \frac{\partial}{\partial \alpha} \frac{\partial}{\partial \beta^{\prime}}-\lambda \frac{\partial}{\partial \beta} \frac{\partial}{\partial \alpha^{\prime}}\right) X^{\mu}(\xi) X^{\nu}\left(\xi^{\prime}\right)\right|_{\xi^{\prime}=\xi} .
$$

For $\lambda=2 \pi / N$, Fairlie, Fletcher, \& Zachos 1989 demonstrate that the Moyal Bracket is essentially equivalent to the commutator of $\mathrm{SU}(N)$ matrices - or subalgebras of $\mathrm{SU}(N)$, depending on the topology of the corresponding membrane surface involved in the Fourier-transform of the color indices [Fairlie, Fletcher, \& Zachos 1990; Kim \& Rey 1997].

In the limit $\lambda \rightarrow 0$, the Moyal Bracket goes to the PB, i.e. $\lambda$ may be thought of as $\hbar$. Thus, when extremely high Fourier modes are ignored, PBs are seen to represent the infinite $N$ limit. This type of identification was first noted without benefit of the MB construction by Hoppe 1982 on a spherical membrane surface; the foregoing MB limit argument was first formulated on the torus [Fairlie, Fletcher, \& Zachos 1989], but readily extends to other topologies [Fairlie, Fletcher, \& Zachos 1989; Fairlie, Fletcher, \& Zachos 1990; Kim \& Rey 1997]. 


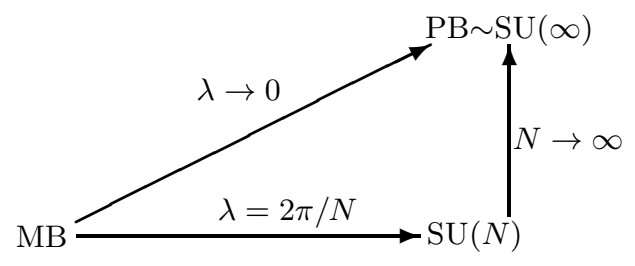

It would suffice to simply treat these most general Moyal Brackets; but their technical manipulations are sometimes more involved and less familiar, so that we also cover the matrix commutator and PB special cases as well, redundancy outweighed by pedagogy.

Floratos et al. 1989 utilize the abovementioned identification of $\mathrm{SU}(\infty)$ with $S D$ iff on a 2 -sphere, to take the large $N$ limit of $\mathrm{SU}(N)$ gauge theory and produce membranes. This procedure was found to be more transparent on the torus [Fairlie, Fletcher, \& Zachos 1990]: the Lie algebra indices Fourier-conjugate to surface coordinates, and the fields are rescaled Fourier transforms of the original $\mathrm{SU}(N)$ fields. The group composition rule for them is given by the PBs and the group trace by surface integration,

$$
\begin{gathered}
{\left[A_{\mu}, A_{\nu}\right] \mapsto\left\{a_{\mu}, a_{\nu}\right\} ;} \\
F_{\mu \nu}=\partial_{\mu} A_{\nu}-\partial_{\nu} A_{\mu}+\left[A_{\mu}, A_{\nu}\right] \mapsto f_{\mu \nu}(\alpha, \beta)=\partial_{\mu} a_{\nu}-\partial_{\nu} a_{\mu}+\left\{a_{\mu}, a_{\nu}\right\} ; \\
\operatorname{Tr} F^{\mu \nu} F_{\mu \nu} \mapsto-\frac{N^{3}}{64 \pi^{4}} \int d \alpha d \beta f^{\mu \nu}(\alpha, \beta) f_{\mu \nu}(\alpha, \beta) .
\end{gathered}
$$

But the large $N$ limit need not really be taken to produce sheet actions. The Lagrangian with the Moyal Bracket supplanting the Poisson Bracket is itself a gauge-invariant theory, provided that the gauge transformation also involves the Moyal instead of the Poisson Bracket:

$$
\delta a_{\mu}=\partial_{\mu} \Lambda-\left\{\left\{\Lambda, a_{\mu}\right\}\right\},
$$

and hence, by virtue of the Jacobi identity,

$$
\delta f_{\mu \nu}=-\left\{\left\{\Lambda, f_{\mu \nu}\right\}\right\} .
$$

Color invariance then follows,

$$
\delta \int d \alpha d \beta f^{\mu \nu} f_{\mu \nu}=-2 \int d \alpha d \beta f^{\mu \nu}\left\{\left\{\Lambda, f_{\mu \nu}\right\}\right\}=0 .
$$

The relevant manipulations are specified in Fairlie, Fletcher, \& Zachos 1990: the last equality is evident by integrations by parts, where the surface term is discarded - or nonexistent if the color membrane surface is closed ${ }^{1}$. For $\lambda=$ $2 \pi / N$, this is equivalent to a conventional $\mathrm{SU}(N)$ commutator gauge theory.

Consider, with Hoppe 1982, the SU( $\infty)$ Yang-Mills lagrangian; and trivialize all space dependence (through dimensional reduction), leaving only time

\footnotetext{
${ }^{1}$ But note this topological term may be nontrivial for D-membranes.
} 
dependence, while preserving all the color-Fourier-space (membrane coordinates $\xi=\alpha, \beta)$ dependence of the gauge fields, which are now denoted $X^{\mu}(t, \alpha, \beta)$. Fix the gauge to $X^{0}=0$, and consider $\mu, \nu$ to henceforth only range over spacelike values.

The Yang-Mills lagrangian density now reduces to the bosonic membrane lagrangian density

$$
\mathcal{L}_{P B}=\frac{1}{2}\left(\partial_{t} X^{\mu}\right)^{2}-\frac{1}{4}\left\{X^{\mu}, X^{\nu}\right\}^{2} .
$$

The PB is also the determinant of the tangents to the membrane, so that the conventional "potential term" was identified in Fairlie, Fletcher, \& Zachos 1990 as the Schild-Eguchi string lagrangian density [Schild 1977] (sheet area squared instead of area), $\left\{X_{\mu}, X_{\nu}\right\}\left\{X_{\mu}, X_{\nu}\right\}$. It can be seen that the equations of motion of such a string action contain those of Nambu's action.

Note that, fixing the gauge $X_{0}=0$ preserves the global color invariance, i.e. with a time-independent parameter $\Lambda(\alpha, \beta)$. The action is then invariant under

$$
\delta X^{\mu}=\left\{\Lambda, X^{\mu}\right\} .
$$

By Noether's theorem, this implies the time invariance of the color charge,

$$
\mathcal{Q}_{\Lambda}=\int d \alpha d \beta \Lambda(\alpha, \beta)\left\{\partial_{t} X^{\mu}, X^{\mu}\right\}
$$

The same also works for the Moyal case [Fairlie, Fletcher, \& Zachos 1990]. The corresponding Moyal Schild-Eguchi term was utilized to yield a "star-productmembrane" [Hoppe 1990],

$$
\mathcal{L}_{M B}=\frac{1}{2}\left(\partial_{t} X^{\mu}\right)^{2}-\frac{1}{4}\left\{\left\{X^{\mu}, X^{\nu}\right\}\right\}^{2},
$$

invariant under

$$
\delta X^{\mu}=\left\{\left\{\Lambda, X^{\mu}\right\}\right\} .
$$

As argued, this includes the commutator case (QM matrix model),

$$
\mathcal{L}_{C}=\frac{1}{2}\left(\partial_{t} X^{\mu}\right)^{2}-\frac{1}{4}\left[X^{\mu}, X^{\nu}\right]^{2},
$$

invariant under

$$
\delta X^{\mu}=\left[\Lambda, X^{\mu}\right] .
$$




\section{Cubic Terms for 3 Dimensions}

By suitable integrations by parts, it is straightforward to check that the cubic terms $(1,2,3)$ in the respective actions, $\int d t d \alpha d \beta \mathcal{L}$, are 3-rotational invariant, as well as time-translation invariant and translation symmetric. They are also global color invariant, as specified above.

Now, further consider a plain mass term in the action ${ }^{2}$,

$$
\mathcal{L}_{3 d P B}=\frac{1}{2}\left(\partial_{t} X^{\mu}\right)^{2}-\frac{1}{4}\left\{X^{\mu}, X^{\nu}\right\}^{2}-\frac{m}{2} \epsilon^{\mu \nu \kappa} X^{\mu}\left\{X^{\nu}, X^{\kappa}\right\}-\frac{m^{2}}{2}\left(X^{\mu}\right)^{2}
$$

The second order equation of motion,

$$
\partial_{t}^{2} X^{\mu}=-m^{2} X^{\mu}-\frac{3 m}{2} \epsilon^{\mu \nu \kappa}\left\{X^{\nu}, X^{\kappa}\right\}-\left\{X^{\nu},\left\{X^{\mu}, X^{\nu}\right\}\right\}
$$

follows not only from extremizing the action, but also results from a first-order equation of the Nahm (self-dual) type [Nahm 1983], albeit complex,

$$
\partial_{t} X^{\mu}=i m X^{\mu}+\frac{i}{2} \epsilon^{\mu \nu \kappa}\left\{X^{\nu}, X^{\kappa}\right\}
$$

These equations hold for PBs, as well as for MBs and matrix commutators.

For solutions of this first-order equation, the conserved energy vanishes. In general, however, such solutions are not real, and do not provide absolute minima for the action - the reader may consider the simple harmonic oscillator to illustrate the point. Nonetheless, the lagrangian density can be expressed as a sum of evocative squares with positive relative signs, since the potential in (20) is such a sum,

$$
\mathcal{L}_{3 d P B}=\frac{1}{2}\left(\partial_{t} X^{\mu}\right)^{2}-\frac{1}{2}\left(m X^{\mu}+\frac{1}{2} \epsilon^{\mu \nu \kappa}\left\{X^{\nu}, X^{\kappa}\right\}\right)^{2} .
$$

By integration by parts in the action $\int d t d \alpha d \beta \mathcal{L}_{3 d P B}$, the lagrangian density itself can then be altered to

$$
\mathcal{L}_{3 d P B} \cong-\frac{1}{2}\left(i \partial_{t} X^{\mu}+m X^{\mu}+\frac{1}{2} \epsilon^{\mu \nu \kappa}\left\{X^{\nu}, X^{\kappa}\right\}\right)^{2},
$$

just like the conventional bosonic membrane lagrangian density ${ }^{3}$. Naturally, the complex-conjugate versions of the above are equally valid.

\footnotetext{
${ }^{2}$ N.B. Of the type that may arise as a remnant of space gradients in compactified dimensions.

3 The congruence symbol, $\cong$, denotes equivalence up to surface terms, which, e.g., vanish for a closed surface; again, consideration of D-membranes would proceed separately.
} 


\section{Vacuum Configurations and their Symmetry}

The minimum of the conventional matrix membrane trough potential favors alignment of the dynamical variables $X^{\mu}$ s. The mass parameter introduced above parameterizes a partial trough symmetry breaking 4 , but does not lift "dilation" invariance, seen as follows.

The static ( $t$-independent) minima for the action (vacuum configurations) are solutions of

$$
m X^{\mu}+\frac{1}{2} \epsilon^{\mu \nu \kappa}\left\{X^{\nu}, X^{\kappa}\right\}=0 .
$$

The previously considered case, $m=0$, is easily solved by "color-parallel" configurations. But for $m \neq 0$, the static solutions must lie on a 2 -sphere, since from the previous equation

$$
X^{\mu} \frac{\partial X^{\mu}}{\partial \alpha}=0=X^{\mu} \frac{\partial X^{\mu}}{\partial \beta},
$$

so

$$
X^{\mu} X^{\mu}=R^{2}
$$

an unspecified constant ${ }^{5}$. However, from (25), note that both $m$ and also $R$, the scale of the $X^{\mu}$ s, can be absorbed in the membrane coordinates $\xi$ and will not be specified by the solution of (25).

Indeed, solving for one coordinate component on this sphere, say

$$
X(Y, Z)= \pm \sqrt{R^{2}-Y^{2}-Z^{2}},
$$

reduces the three equations (25) to one. Namely,

$$
\{Z, Y\}=m \sqrt{R^{2}-Y^{2}-Z^{2}},
$$

on the positive $X$ branch ( $m \mapsto-m$ on the negative $X$ branch). This last equation is solved by

$$
Z=\alpha, \quad Y=\sqrt{R^{2}-\alpha^{2}} \sin (m \beta) .
$$

One can then interpret $m \beta$ as the usual azimuthal angle around the $Z$-axis. Hence, $-\pi / 2 \leq m \beta \leq \pi / 2$ and $-R \leq \alpha \leq R$ covers the $X \geq 0$ hemisphere completely. The other hemisphere is covered completely by the negative $X$ branch. Since $R$ is not fixed, it amounts to an unlifted residual trough dilation degeneracy.

All static solutions are connected to this explicit one by rescaling $R$ and exploiting the equation's area-preserving diffeomorphism invariance for $\xi=(\alpha, \beta)$.

\footnotetext{
${ }^{4}$ Curtright \& McCarty (1989) have considered a system equivalent to the limit of constant $X^{3}=$ in this model.

${ }^{5}$ Hence $\epsilon^{\mu \nu \kappa} X^{\mu}\left\{X^{\nu}, X^{\kappa}\right\}=-2 m R^{2}$.
} 


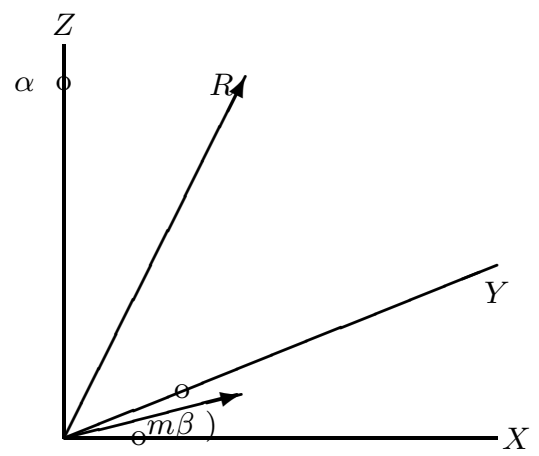

\section{Nahm's Equation and its Lax Pairs}

The first order equation, (22), simplifies upon changing variables to

$$
\tau=\frac{\mathrm{e}^{i m t}}{m}, \quad X^{\mu}=\mathrm{e}^{i m t} Y^{\mu},
$$

and reduces to the conventional PB version [Ward 1990,Floratos \& Leontaris 1989] of Nahm's equation [Nahm 1983],

$$
\partial_{\tau} Y^{\mu}=\frac{1}{2} \epsilon^{\mu \nu \kappa}\left\{Y^{\nu}, Y^{\kappa}\right\} .
$$

This one does have real solutions, and can be linearized by Ward's transformation [Ward 1990]. However, the action (23) does not reduce to the conventional one upon these transformations ${ }^{6}$.

Moreover, note

$$
\begin{gathered}
\partial_{\tau} Y^{\mu} \partial_{\tau} Y^{\mu}=\frac{\partial\left(Y^{1}, Y^{2}, Y^{3}\right)}{\partial(\tau, \alpha, \beta)}, \\
\partial_{\tau} Y^{\mu} \partial_{\xi} Y^{\mu}=0 .
\end{gathered}
$$

One may further utilize the cube root of unity, $\omega=\exp (2 \pi i / 3)$, to recast $^{7}$ $(32)$,

$$
\begin{gathered}
L \equiv \omega Y^{1}+\omega^{2} Y^{2}+Y^{3}, \quad \bar{L}=\omega^{2} Y^{1}+\omega Y^{2}+Y^{3}, \quad M \equiv Y^{1}+Y^{2}+Y^{3}, \\
\omega(\omega-1) \partial_{\tau} L=\{M, L\}, \quad \omega(\omega-1) \partial_{\tau} \bar{L}=-\{M, \bar{L}\}, \\
\omega(\omega-1) \partial_{\tau} M=\{L, \bar{L}\},
\end{gathered}
$$

which thus yields an infinite number of complex time-invariants,

$$
Q_{n}=\int d \alpha d \beta L^{n}
$$

\footnotetext{
${ }^{6}$ Likewise, the second order equations of motion only reduce to $\partial_{\tau}^{2} Y^{\mu}=$ $\left\{Y^{\nu},\left\{Y^{\mu}, Y^{\nu}\right\}\right\}+\frac{3}{\tau}\left(\partial_{\tau} Y^{\mu}-\frac{\epsilon^{\mu \nu \kappa}}{2}\left\{Y^{\nu}, Y^{\kappa}\right\}\right)$.

7 N.B. $\omega(\omega-1)$ is pure imaginary.
} 
for arbitrary integer power $n$, as the time derivative of the integrand is a surface term. (This is in complete analogy with the standard case of commutators.) The link to classical integrability is discussed next.

Equations $(36,37)$ amount to one complex and one real equation, but these are known to be further capable of compacting into just one by virtue of an arbitrary real spectral parameter $\zeta$, e.g. as introduced in Floratos \& Leontaris 1989:

$$
\begin{gathered}
H \equiv \frac{i}{\sqrt{2} \omega(\omega-1)}\left(\zeta L-\frac{\bar{L}}{\zeta}\right), \quad K \equiv i \sqrt{2} M+\zeta L+\frac{\bar{L}}{\zeta}, \\
\partial_{\tau} K=\{H, K\} .
\end{gathered}
$$

Likewise, this Lax pair ${ }^{8}$, analogous to Hitchin 1983, leads to a one-parameter family of time-invariants ${ }^{9}$,

$$
\mathcal{Q}_{n}(\zeta)=\int d \alpha d \beta K^{n}
$$

conserved for all $n$ and $\zeta$.

It also yields the usual Lax isospectral flow. PBs (and, mutatis mutandis, MBs) can be recast into commutators of suitable associative operators

$$
\mathcal{K} \equiv \nabla K \times \nabla, \quad \mathcal{H} \equiv \nabla H \times \nabla, \quad \nabla \equiv\left(\frac{\partial}{\partial \alpha}, \frac{\partial}{\partial \beta}\right)
$$

such that:

$$
\partial_{\tau} \mathcal{K}=\mathcal{H} \mathcal{K}-\mathcal{K H}
$$

As a consequence, the spectrum of $\mathcal{K}$ is preserved upon time evolution by the (pure imaginary) $\mathcal{H}$ :

$$
\partial_{\tau} \psi=\mathcal{H} \psi
$$

since time-differentiating

$$
\mathcal{K} \psi=\lambda \psi
$$

and applying (43) yields

$$
\left(\partial_{\tau} \lambda\right) \psi=\left(\partial_{\tau} \mathcal{K}\right) \psi+\mathcal{K} \partial_{\tau} \psi-\lambda \partial_{\tau} \psi=0 .
$$

This isospectral flow then provides integrability for (22), as in the case of the matrix commutator Nahm equation.

\footnotetext{
${ }^{8}$ Note the wave solutions $H=\alpha, K=f(\beta+\tau)$.

${ }^{9}$ L. Dickey calls our attention to the parameter introduced in the Lax pair for the generalized Euler equations by S. Manakov, Funct Anal Appl 10 (1976) 328, which parallels that in Hitchin's Lax pair for the Nahm equations.
} 
The discussion so far also carries over to plain matrix commutators or Moyal Brackets as well, with suitable adaptations for the associative operators involved, e.g. a $\star$-product structure [Fairlie, Fletcher, \& Zachos 1989; Hoppe 1990],

$$
\left.\mathbb{K} \equiv \frac{1}{2 i \lambda} \mathrm{e}^{i \lambda \nabla^{\prime} \times \nabla} K\left(\xi^{\prime}\right)\right|_{\xi^{\prime}=\xi} .
$$

For the rest of this section, we shall restrict our attention to the PB case only. Ward 1990 has solved the PB Nahm equation (32) implicitly through twistor linearization. Another solution procedure for (32) may be found by interchanging the rôles of dependent and independent variables: the equations then take the dual form

$$
\frac{\partial \tau}{\partial Y^{1}}=\frac{\partial \alpha}{\partial Y^{2}} \frac{\partial \beta}{\partial Y^{3}}-\frac{\partial \alpha}{\partial Y^{3}} \frac{\partial \beta}{\partial Y^{2}},
$$

together with cyclic permutations, i.e.

$$
\partial_{\mu} \tau=\epsilon^{\mu \nu \rho} \partial_{\nu} \alpha \partial_{\rho} \beta
$$

Cross-differentiation produces integrability conditions

$$
\partial_{\mu}\left(\partial_{\kappa} \alpha \partial_{\mu} \beta-\partial_{\mu} \alpha \partial_{\kappa} \beta\right)=0 .
$$

Another evident consistency condition is

$$
\partial^{2} \tau=0 \text {. }
$$

In fact, any harmonic function $\tau\left(Y^{1}, Y^{2}, Y^{3}\right)$, readily yields $\alpha, \beta$. Euler appreciated (1770) that any continuous differentiable divergenceless vector field may be represented locally as a cross product of two gradients [Ericksen 1960]. The problem of solving the inverse Nahm equation (49) then reduces to Euler's construction, given an arbitrary harmonic function $\tau$.

To determine scalar fields $\alpha$ and $\beta$, note that

$$
\nabla \alpha \cdot \nabla \tau=0=\nabla \beta \cdot \nabla \tau,
$$

so that $\nabla \tau$ lies on surfaces of constant $\alpha$ and $\beta$. One may then choose the surface of constant $\alpha$ through $\nabla \tau$ arbitrarily, and further choose an arbitrary continuous vector field $\mathbf{V}$ s.t. $\mathbf{V} \cdot \nabla \alpha \neq 0$. One may thus integrate on the constant- $\alpha$ surface to obtain $\beta$ :

$$
\beta=\int d \mathbf{Y} \cdot \frac{\nabla \tau \times \mathbf{V}}{\mathbf{V} \cdot \nabla \alpha} .
$$

Evidently, solutions of these dual equations

$$
\partial_{\mu} f=\epsilon^{\mu \nu \rho} \partial_{\nu} g \partial_{\rho} h
$$

for $f\left(Y^{1}, Y^{2}, Y^{3}\right)$ produce constants of the motion $\int d^{2} \xi f$, beyond those already found by the Lax procedure, for the original equation (32), in illustration of a 
phenomenon noted in Fairlie \& Strachan (1996), as it is straightforward to verify that

$$
\frac{\mathrm{d} f}{\mathrm{~d} \tau}=\{g, h\}
$$

From the foregoing discussion, $f$ need only solve Laplace's equation (51): any harmonic function $f\left(Y^{1}, Y^{2}, Y^{3}\right)$ yields a conserved density for (32) by also satisfying (54). To belabor the point, by virtue of Helmholtz's theorem, a divergenceless 3 -vector $\nabla f$ is representable as a curl of another vector $\mathbf{A}$. On the other hand, an arbitrary 3 -vector can also be represented in terms of three scalars ("Monge potentials") by means of the non-unique "Clebsch decomposition" of that vector as $\mathbf{A}=g \nabla h+\nabla u$, in a trivial extension of Euler's construction just outlined [Ericksen 1960].

\section{Membrane Embedding in 7 Dimensions}

Remarkably, the same type of cubic interaction term may also be introduced for a membrane embedded in 7 space dimensions. An antisymmetric, self-dual 4-tensor in 8 dimensions, $f_{\mu \nu \rho \sigma}$ was invoked by Corrigan et al. 1982 as an 8dimensional analog of the 4-dimensional fully antisymmetric tensor $\epsilon_{\mu \nu \rho \sigma}$. Some useful technology for the manipulation of this tensor (which has 35 nonzero components, is linked to Cayley's octonionic structure constants, and is invariant under a particular $S O(7)$ subgroup of $S O(8)$ ) can be found in Dündarer et al. (1984); in particular, the identity

$$
f^{0 \mu \nu \kappa} f^{0 \mu \lambda \rho}=f^{\nu \kappa \lambda \rho}+\delta^{\nu \lambda} \delta^{\kappa \rho}-\delta^{\kappa \lambda} \delta^{\nu \rho} .
$$

By analogy with (32), we postulate a first-order equation ${ }^{10}$,

$$
\partial_{\tau} Y^{\mu}-\frac{f^{0 \mu \nu \kappa}}{2}\left\{Y^{\nu}, Y^{\kappa}\right\}=0 .
$$

The indices run from $\mu=1$ to $\mu=7$, since we are working in a gauge where $Y^{0}=0$. The second order equation arising from iteration of (57), by virtue of the above identity, as well as the Jacobi identity, is

$$
\partial_{\tau \tau} Y^{\mu}=-\left\{\left\{Y^{\mu}, Y^{\nu}\right\}, Y^{\nu}\right\} .
$$

This arises from the lagrangian density

$$
\mathcal{L}_{7 d P B}=\frac{1}{2}\left(\partial_{\tau} Y^{\mu}\right)^{2}+\frac{1}{4}\left\{Y^{\mu}, Y^{\nu}\right\}^{2} .
$$

As in the 3-dimensional case, this action reduces to a sum of squares with positive relative signs, up to a mere surface term,

$$
\frac{1}{2}\left(\partial_{\tau} Y^{\mu}-\frac{f^{0 \mu \nu \kappa}}{2}\left\{Y^{\nu}, Y^{\kappa}\right\}\right)^{2}=\mathcal{L}_{7 d P B}-f^{0 \mu \nu \kappa} \partial_{\tau} Y^{\mu} \partial_{\alpha} Y^{\nu} \partial_{\beta} Y^{\kappa} \cong \mathcal{L}_{7 d P B} .
$$

\footnotetext{
${ }^{10}$ At present, we are not in a position to comment on its integrability or lack thereof.
} 
In this lagrangian density, apparent extra terms $f^{\mu \nu \rho \kappa}\left\{Y^{\mu}, Y^{\nu}\right\}\left\{Y^{\rho}, Y^{\kappa}\right\}$ induced by the identity (56) have, in fact, vanished, by virtue of the identity,

$$
\{f, g\}\{h, k\}+\{f, h\}\{k, g\}+\{f, k\}\{g, h\} \equiv 0,
$$

which holds for Poisson Brackets on a 2-dimensional phase-space-but not for matrix commutators nor Moyal Brackets ${ }^{11}$. This cancellation works at the level of the lagrangian density for the PB case.

However, note that even for ordinary matrices the corresponding term would vanish in the traced lagrangian, by the cyclicity of the trace pitted against full antisymmetry,

$$
f^{\mu \nu \kappa \rho} \operatorname{Tr} X^{\mu} X^{\nu} X^{\kappa} X^{\rho}=0 .
$$

Likewise, the corresponding interaction for Moyal Brackets,

$$
f^{\mu \nu \kappa \rho} \int d^{2} \xi\left\{\left\{X^{\mu}, X^{\nu}\right\}\right\}\left\{\left\{X^{\kappa}, X^{\rho}\right\}\right\},
$$

is forced by associativity to reduce to a surface term, vanishing unless there are contributions from surface boundaries or D-membrane topological numbers involved. (Shortcuts for the manipulation of such expressions underlain by $\star$ products can be found, e.g., in Hoppe 1990.) The cross terms involving time derivatives are expressible as divergences, as in the 3 -dimensional case, and hence may give rise to topological contributions.

As a result, (57) is the Bogomol'nyi minimum of the action of (59) with the bottomless potential.

As in the case of 3-space, the conventional membrane signs can now be considered (for energy bounded below), and a symmetry breaking term $m$ introduced, to yield

$$
\mathcal{L}_{7 d P B} \cong-\frac{1}{2}\left(-i \partial_{t} X^{\mu}+m X^{\mu}+\frac{f^{0 \mu \nu \kappa}}{2}\left\{X^{\nu}, X^{\kappa}\right\}\right)^{2} .
$$

This model likewise has 7-space rotational invariance, and its vacuum configurations are, correspondingly, 2-surfaces lying on the spatial 6-sphere embedded in 7-space: $X^{\mu} X^{\mu}=R^{2}$. But, in addition, because of (56), these surfaces on the sphere also satisfy the trilinear constraint

$$
f^{\lambda \mu \nu \kappa} X^{\mu}\left\{X^{\nu}, X^{\kappa}\right\}=0
$$

for $\lambda \neq 0$. (For $\lambda=0$ this trilinear is $-2 m R^{2}$.)

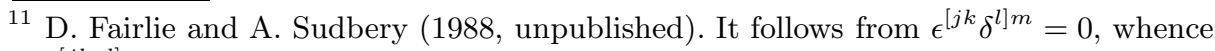
$\epsilon^{[j k} \epsilon^{l] m}=0$ for these membrane symplectic coordinates. 


\section{References}

Banks, T., Fischler, W., Shenker, S., and Susskind, L. (1997): Phys Rev D55 5112

Biran, B., Floratos, E., and Savvidi, G. (1987): Phys Lett B198 329

Collins, P., and Tucker, R. (1976): Nucl Phys B112 150

Corrigan, E., Devchand, C., Fairlie, D., and Nuyts, J. (1982): Nucl Phys B214 452

Curtright, T., Fairlie, D., and Zachos, C. (1997): Phys Lett B405 37

Curtright, T. and McCarty, T. (1989): ICTP'89 talk (unpublished),

(http://phyvax.ir.miami.edu:8001/curtright/ictp89.html); and the latter's University of Florida Thesis (unpublished)

Curtright, T., and Zachos, C. (1995): in PASCOS'94, K. C. Wali (ed), (World Scientific), pp 381-390, (hep-th/9407044)

Dündarer, R., Gürsey, F., and Tze, C-H. (1984): J Math Phys 251496

Ericksen, J. (1960): Appendices 32, 34, \& 35 in Vol. III/1 of the Encyclopedia of Physics (Handbuch der Physik), S. Flügge (ed), (Springer, Berlin) pp 822-829

Fairlie, D. (1997): hep-th/9707190

Fairlie, D., Fletcher, P., and Zachos, C. (1989): Phys Lett B218 203;

D. Fairlie and C. Zachos, Phys Lett B224 (1989) 101

Fairlie, D., Fletcher, P., and Zachos, C. (1990): J Math Phys 311088

Fairlie, D., and Strachan, I. (1996): Physica D90 1

Floratos, E., (1989): Phys Lett B228 335

Floratos, E., Iliopoulos, J., and Tiktopoulos, G. (1989): Phys Lett B217 285; also see

B. de Wit, J. Hoppe, and H. Nicolai, Nucl Phys B305 [FS23] (1988) 545;

J. Hoppe, Int J Mod Phys A4 (1989) 5235;

E. Bergshoeff, E. Sezgin, Y. Tanii, and P. Townsend, Ann Phys 199 (1990) 340

Floratos, E., and Leontaris, G. (1989): Phys Lett B223 153

Hitchin, M. (1983): Comm Math Phys 89145

Hoppe, J. (1982): M.I.T. Ph.D. Thesis; also in Elem Part Res J (Kyoto) 83 no.3 $(1989 / 90)$

Hoppe, J. (1990): Phys Lett B250 44

Kim, N., and Rey, S-J. (1997): hep-th/9701139

Matinyan, S., Savvidy, G., and Ter-Arutunian Savvidy, N. (1981): Sov Phys JETP 53 421; JETP Lett 34 (1981) 590;

S. Matinyan, E. Prokhorenko, and G. Savvidy, Nucl Phys B298 (1988) 414

Moyal, J. (1949): Proc Camb Phil Soc 45 99; further see

J. Vey, Comment Math Helvetici 50 (1975) 412;

T. Jordan and E. Sudarshan, Rev Mod Phys 33 (1961) 515;

D. Fairlie, Proc Camb Phil Soc 60 (1964) 581;

D. Fairlie and C. Manogue, J Phys A24 (1991) 3807

Nahm, W. (1983): in Group Theoretical Methods in Physics: XIth International Colloquium, Istanbul, 1982, M. Serdaroğlu and E. Ínönü (eds), (Springer Lecture Notes in Physics 180, Berlin) pp 456-466

Plebański, J., Przanowski, M., and García-Compeán, H. (1996): Mod Phys Lett A11 663

Schild, A. (1977): Phys Rev D16 1722; aso see

T. Eguchi, Phys Rev Lett 44 (1980) 126

Ward, R. (1990): Phys Lett B234 81

Zaikov, R. (1991): Phys Lett B266 303 
14 Cosmas Zachos, David Fairlie, and Thomas Curtright

Zakharov, V., and Mikhailov, A. (1978): Sov Phys JETP 47 1017;

T. Curtright and C. Zachos, Phys Rev D49 (1994) 5408 